\title{
Revisión de la gramática emergente: lenguajes, jóvenes y mediaciones $\mathrm{TIC}^{\star}$
}

\author{
Mireya Barón Pulido* \\ Recibido: 18 de julio \\ Aprobado: 17 de noviembre
}

\begin{abstract}
Resumen
El presente artículo de revisión recoge dos miradas: la primera hace alusión a la caracterización de las nuevas tecnologías de la Información-Comunicación NTIC y de las mediaciones en el contexto educativo universitario; la segunda da a conocer la tendencia de resultados de investigación de las NTIC en América Latina y Europa en los últimos 5 años. La documentación consultada recoge estudios tanto de orden cualitativo como cuantitativo; pertenece a bases de datos indexadas, y los enfoques involucran desde el contexto psicológico, hasta el educativo, el comunicativo y el antropológico-cultural, en los que se reconoce la existencia de la Generación NET ("Net Generation": Oblinger, 2005; Nativos Digitales: Piscitelli, 2009; Multitasking Generation; Gruffat, 2004). Producto de esta revisión documental, surge el diseño de la Investigación "Semiosis en sistemas sígnicos y mediaciones TIC en población estudiantil Politécnico Grancolombiano-Colombia", inscrito ante Colciencias, en el 2011.
\end{abstract}

Palabras clave: Ntic, mediaciones, estudios.

* El proyecto de investigación del cual es producto este artículo, ha sido financiado por la Fundación Politécnico Grancolombiano, mediante el contrato de investigación N²011-FMCA-CEC-TC-13.

** Docente Investigadora del Departamento Académico de Comunicación Social-Periodismo de la. Facultad de Mercadeo, Comunicación y Artes. Institución Universitaria Politécnico Grancolombiano. Magíster en Lingüística Española e Hispanoamericana. Líder del Grupo de Investigación CEC Comunicación Estratégica y Creativa. Correo electrónico: mbaronpu@poli. edu.co 


\title{
Review of the Emerging Grammar: Languages, Youth, and ICT Mediations
}

\begin{abstract}
This review article is based on two points of view: the first one is related to characterization of New Information and Communication Technologies (NICT) and the mediations in the university educational context; the second one show a tendency NICT research results have had in Latin America and Europe during the last 5 years. Documents consulted involve both qualitative and quantitative studies; documents were taken from indexed databases and approaches involve the psychological context and the educational, communicational, and anthropological-cultural contexts recognizing the existence of the NET Generation ("Net Generation": Oblinger, 2005; Nativos Digitales: Piscitelli, 2009; Multitasking Generation; Gruffat, 2004). Product of this documentary review, the research design emerges: "Semiosis en sistemas sígnicos y mediaciones TIC en población estudiantil Politécnico Grancolombiano-Colombia" registered with COLCIENCIAS in year 2011.
\end{abstract}

Key words: NTIC; mediations; studies. 
Revisión de la gramatica emergente: lenguajes, jovenes y mediaciones tic *

\section{Las NTIC, mucho más que aparatos}

El individuo de la sociedad del siglo XXI construye de manera permanente sentido a partir de los diversos y emergentes contextos que las coordenadas geo-espaciales e históricas le posibilitan. Junto al tiempo sincrónico (on line), fluye el tiempo asincrónico (offline), conceptos neurálgicos en el día a día de las actividades diarias y académicas en los jóvenes universitarios de hoy.

El contexto de las mediaciones, es decir, el de las redes de sentido, el de las acciones mediadas por tecnología y por bienes simbólicos, entre otros, es ya una constante en el modus vivendi del ciudadano promedio.

La internet es la principal Nueva Tecnología de la Información y de la Comunicación, NTIC, a la que estamos expuestos, advierte el profesor mexicano Méndez. La llamada generación de INTERNET es joven, por cuanto la tecnología nació en la década de 1970 (Méndez, 2005) y a partir de este escenario, en diversas regiones del mundo, surgen diversas clases de mediaciones.

El ecuatoriano Serafin Ilvay explica desde el clásico concepto griego " technè " (fabricación), pasando por la técnica (método), la tecnología (aplicación), el concepto de las NTIC las caracteriza así: "Las nuevas tecnologías tienen la característica de revolucionar lo establecido, la mezcla ya explicada de la ciencia, la técnica y la sociedad, marcan hitos en el desarrollo de una u otra área o varias al mismo tiempo" (Ilvay, 2000). Sobre estos lineamientos se desarrolla el artículo de revisión, el cual intenta argumentar, a través de una pesquisa detallada, las acepciones e impacto que sugiere el escenario de las MEDIACIONES, de los LENGUAJES y de las TIC (Tecnologías de la Información y Comunicación) en la comunidad universitaria.

La caracterización de este entorno nos exige tener presentes a las TIC en sus contextos sociohistóricos en los que se producen; por ello se dan algunas definiciones (Figura 1)

Figura 1. Hacia una caracterización de las TIC

\begin{tabular}{|l|l|}
\hline \multicolumn{1}{|c|}{ Autor (a) } & \multicolumn{1}{c|}{ Caracterización de TIC } \\
\hline Manuel Castells & $\begin{array}{l}\text { "Comprenden una serie de aplicaciones de descubrimiento científico cuyo núcleo central } \\
\text { consiste en una capacidad cada vez mayor de tratamiento de la información" (Castells, 1986) }\end{array}$ \\
\hline $\begin{array}{l}\text { Delia Crovi } \\
\text { Druetta }\end{array}$ & $\begin{array}{l}\text { "Reemplazan el sistema analógico por el digital, con el que inauguran, en el área de las } \\
\text { comunicaciones, nuevos sistemas de transmisión a distancia" (Crovi, 2000) }\end{array}$ \\
\hline Jimmy Rosario & $\begin{array}{l}\text { "Conjunto de tecnologías que permiten la adquisición, producción, almacenamiento, trata } \\
\text { miento, comunicación, registro y presentación de informaciones, en forma de voz, imágenes } \\
\text { y datos contenidos en señales de naturaleza acústica, óptica o electromagnética. Las TIC incluyen la } \\
\text { electrónica como tecnología base que soporta el desarrollo de las telecomunicaciones, } \\
\text { la informática y el audiovisual" (Rosario, J, 2006) }\end{array}$ \\
\hline $\begin{array}{l}\text { "Son aquellas herramientas computacionales e informáticas que procesan, almacenan, } \\
\text { sintetizan, recuperan y presentan información representada de la más variada forma. Es } \\
\text { un conjunto de herramientas, soportes y canales para el tratamiento y acceso a la infor } \\
\text { mación. Constituyen nuevos soportes y canales para dar forma, registrar, almacenar y difundir } \\
\text { contenidos informacionales" (Mociño José,2000) }\end{array}$ \\
$\begin{array}{l}\text { (...) conjunto de procesos y productos derivados de las nuevas herramientas (hardware y } \\
\text { software) soportes de la información y canales de comunicación relacionados con el al- } \\
\text { macenamiento, procesamiento y transmisión digitalizados de la información (Adell, 1997) }\end{array}$ \\
\hline
\end{tabular}

Fuente: autoría propia (La cursiva es de la autora del artículo) 
Lo relevante no es definir las TIC, destacando su rol interactivo con el usuario; lo que demanda la atención, es el conjunto de niveles de CONSTRUCCIÓN DE SENTIDO, DE USO y DE APROPIACIÓN en el actor que interactúa con las TIC: los rasgos de materialización sígnica icónica, textual y sonora; la incursión de una sintaxis por otra; la aparición de morfologías híbridas lingüísticomatemáticas, la prelación de una grafía-fonética y recreaciones gramaticales, entre otros.

Ahora bien, vale la pena reconocer dos componentes de las TIC: Interactividad, sistemas combinados entre sí, respuestas al usuario, y Convergencia, es decir, que aglutinan varios servicios (telefonía, cámara de fotos, reproductor de música, navegador de Internet, agenda, despertador...entre otros). Este fenómeno semiótico -de la significación, de lo simbólico-, conduce a un segundo eje a revisar: MEDIACIÓN, MEDIATIZACIÓN DE LA CULTURA, caracterizado por Thompson como:

"Un proceso de transformaciones profundas e irreversibles en los modos de producir, distribuir y poner en circulación los bienes simbólicos; los tiempos y espacios de producción y recepción se separan, aparecen los llamados mensajes masivos que son aquellos que están destinados a un gran número de personas a las que generalmente se agrupa en torno a características que, desde los lugares de producción y emisión, creen que esos grupos tienen en común" (Thompson, 1998).

En el fluir de este proceso se destacan, a la vez, diversos tipos de mediación, para lo cual acudimos al estudio detallado de la argentina Fainholc. Con la intención de superar el enfoque instrumental de la mediación, en tanto que esta hace parte de la estructura misma de la sociedad del conocimiento, en la actual revisión conceptual no se enfatiza en los aparatos. Se sugiere, en cambio, concebir la mediación, atendiendo lo analizado por Beatriz Fainholc, en relación con los nuevos modos de percepción, recepción, significantes y construcción del lenguaje; nuevas narrativas, escrituras, y nuevas sensibilidades que moldean al sujeto (Fainholc, 2004).

El concepto de mediación, desde el marco histórico del término, evoca, en primer lugar, a Vigotsky, psicólogo que lo caracteriza como "acción mediada". En este marco, apreciemos el siguiente gráfico en el que se destacan 4 tipos de mediación, todas enfocadas en el eje pedagógico: (Figura 2)

Figura 2. Clases de mediación en el contexto educativo

\section{La mediación cultural:}

Compuesta por bienes materiales y simbólicos, entre ellos, el fundamental es el lenguaje Mediación: Tecnológica o Proceso Comunicacional

\section{Mediación semiológica:}

Sistemas simbólicos referidos a:

a) Contenidos (lo que dice)

b) Organización del contenido (cómo se presenta)

c) Uso del contenido (cómo y para qué es empleado)

Apuntando a una relación de PODER

\section{Mediación} Tecnológica:

Mindware que sabe utilizar un Softv/ are y Hardware, es decir, conjunto de habilidades que relaciona el sujeto con SFT/ HDW Hombre en la Cultura Tecnológica 
Resulta valioso analizar en futuras investigaciones la mediación semiológica, ya que es relevante el conocimiento sobre las configuraciones de sentido y de lo representacional en los estudiantes universitarios. En este orden de ideas, vale la pena aclarar que dado que las mediaciones conforman redes de sentidos no sólo contextuales sino intertextuales (Araujo, 2002), es pertinente atender a Fainholc, citando a Araujo, quien advierte que es imposible analizar todas las mediaciones en un acto comunicativo.

En estos términos, y si bien es cierto la práctica escolar académica se asume en tanto proceso cultural, también es cierto que se afronta una realidad de construcción de significantes -formas o imágenes sígnicas gráficas, sonoras, visuales (Dubois, 1998)- que de manera progresiva se han ido acordando a través de consensos de uso mediados por el entorno y la praxis digital.

Esas puestas en común ya permean los bienes simbólicos y los mensajes masivos creados por la generación digital, que remite al concepto de EL JOVEN. De los diversos enfoques que caracterizan al individuo joven, resulta oportuno tener presente que:

"....lo joven no está dado únicamente por una cuestión etaria, sino que es necesario pensar cómo atraviesan a esta categoría cuestiones sociodemográficas, de grupos de pertenencia y de contextos políticos y sociales. Es decir, la categoría de juventud puede variar de acuerdo con las distintas regiones, clases sociales, contextos. ... Se considerarán jóvenes a las personas comprendidas en el rango etario que va de los 18 a los 30 años. Sabemos que es una franja amplia cuando de tecnologías se trata. Los que están cerca de los 30 no pueden incluirse en el grupo de los llamados nativos tecnológicos..." (Racioppe, 2008, p. 5)

En estos términos, resulta valiosa la argumentación antropológica cultural que hace la mexicana
Reguillo: "La sociedad reivindicó la existencia de los niños y los jóvenes como sujetos de derechos y, especialmente, en el caso de los jóvenes, como sujetos de consumo" (Reguillo, 2000, p. 23. La cursiva es de la autora).

La investigación en psicología social crítica (Gil, et al; 2003), se refiere al individuo en esta sociedad de consumo, en la medida que se fragmenta en tantas identidades como en las diversas y variadas situaciones que vive junto a las TIC, es decir la sociedad se encuentra ante una generación en la que "el individuo ya no es la fuente de todas sus determinaciones, sino que es el producto de un dispositivo de prácticas semióticas y materiales (Rose, 1996; Rivero, 2001 y 2002). En la investigación realizada por el grupo de españoles Gil, Feliu y Rivero, se advierte el cliché con que adultos estigmatizan a la generación net, como lo mencionan otros estudiosos; la idea no es agrandar la brecha generacional; la propuesta, sugieren estos españoles, es reconocer cómo se construye una cultura digital entendida como el encuentro alrededor de las TIC. Afrontamos un escenario de nuevas prácticas culturales, donde la(s) identidad (es) del individuo adopta(n) ciertas estéticas, en medio de un tejido de relaciones, sonidos, lenguajes y espacios físicos-virtuales. La identidad surge en medio de la selección de actividades y objetos simbólicos (Feixa, et al, 2002)

Quien fluye en las mediaciones para forjar su identidad es quien crea e inventa SIGNOS; por tanto, no se debe olvidar que los diversos tipos de mediación, emergen de él: "Desde la dimensión del signo como mediador social, Vygotsky expresa que en la creación del mismo, éste es empleado como una herramienta cuyo propósito es esencialmente el de establecer la comunicación a través de las relaciones sociales" (Gutiérrez, 2008: 1)

A partir del signo se empieza a hablar de SEMIOSIS. En términos peirceanos, desde la relación triádica: referente $\mathcal{E}$ signo $\mathcal{E}$ interpretante, hablamos de semiosis: 
".....podemos apuntar que si bien los signos surgen en plano intermental -lo cual supone que no puede existir un signo sin cosa u objeto designado, y que el significado de un signo no es una cualidad intrínseca sino que emerge de un acto interpretativo- entonces el significado no puede considerarse como algo inamovible ni eterno, sino algo cambiante. La cultura, dado su carácter dinámico, complejo y variable en función de sus ideologías, intereses y necesidades, ajustará los signos y sus significados de acuerdo con las representaciones mentales establecidas en la relación comunicativa de los seres humanos" (Gutiérrez, 2008: 4)

Junto a la clasificación que hace Fainholc sobre las mediaciones, resulta valioso citar el estudio español que explica el concepto de MEDIACIÓN SOCIAL-INTERCULTURAL, el cual demanda la actuación de otros dos conceptos: educación intercultural y mediación social. Al respecto, vale la pena destacar: "la importancia de la mediación social en el ámbito educativo, que a pesar de no estar aun claramente definidas sus funciones y roles, sí se reconoce su potencial para fomentar nuevas habilidades y actitudes de negociación, participación y diálogo en contextos de desigualdad, configurando una nueva forma de establecer las relaciones interpersonales entre los diversos agentes educativos y entre ellos y sus destinatarios". (Aguado, 2006)

En este caso se destaca el impacto y afectación que genera el concepto de mediación, superando el simple uso de aparatos tecnológicos. En este estudio europeo se reconoce y se convierte en objeto de estudio otro tipo de mediaciones, para este caso: la mediación socio-cultural; inclusive, el estudio propone la elaboración de documentos y recursos para formación de docentes en el ámbito de la mediación social intercultural, dadas las características etnográficas de algunas regiones del país que han permitido la creación de la ESCUELA DE MEDIACIÓN SOCIAL para migran- tes, la SEMSI (Servicio de Mediación Intercultural de la Comunidad de Madrid).

El concepto de mediación afecta, inclusive hasta llegar a "transgredir", la relación SUJETO / OBJETO. Esta caracterización se aprecia a partir de la mediación tecnológica. En estos términos, advierte el estudio de Torreblanca, al citar al especialista Díaz-B:

"Mediación a través de artefactos tecnológicos educativos.......se refiere a los artefactos culturales, los enmarca dentro de la noción de mediación, apuntando al hecho de que las relaciones entre el sujeto y el objeto solo pueden darse de dos maneras: inmediatas, directas y naturales, o bien, mediadas a través de los artefactos culturales disponibles. En ese sentido, cualquier artefacto cultural está imbuido de significado y valor por existir dentro de un campo de la actividad humana, y ofrece una enorme "potencialidad como instrumento mediacional del funcionamiento cognitivo, como sistema de construcción de significados o de transformación y creación de contenidos culturales" (DiazBarriga, 2005: 4).

Por tanto, es relevante apreciar en contextos y sujetos específicos, los niveles de uso, significación y valor que adquiere este tipo de aparatos tecnológicos (portátiles, telefonía móvil) en sus interlocutores y constructores de sentido. La profesora Frida Díaz hace una recomendación: "Las TIC sólo se perciben como herramientas técnicas o artefactos físicos cuyo empleo facilita o hace más eficientes las formas de acción que ocurrirían de otra manera. Sin embargo, se deja de lado el sentido de herramienta semiótica" (Díaz-Barriga, 2005:6). Precisamente, la mediación tecnológica, per se, es ya una realidad, sin embargo, es en la mediación semiológica, simbólica, en donde yace todo esta nueva relación sujeto, objeto, signo: "implica una forma de razonamiento o argumentación asociada a determinadas creencias, reglas y normativas sociales que determinan a su vez el 
sentido y uso de dicha herramienta" (Díaz-Barriga, 2005: 8).

Al extrapolar las potencialidades y las posibilidades de acceso a la información desde las NTIC, seguramente la ganancia entre sujeto/objeto en todos los entornos sería mayor. Al respecto, Coll destaca los siguientes factores, de cara al aprovechamiento de las NTIC en el contexto educativo: (figura 3)

\section{SUJETOS Y OBJETOS EN LA MEDIACIÓN}

Generación net (Oblinger,2005), Generación multimedia (Morduchowicz, 2008), Nativos digitales (Piscitelli, 2009), Generación M mediamultitasking (Gruffat, 2004) son algunos de los referentes para caracterizar al sujeto actor de este contexto cibercomunicativo.
El maestro argentino Piscitelli (2010) se refiere a la generación digital como los individuos nacidos después de los 80 , que viven en la pantalla y son masificadores del uso de computadores y móviles; amantes de la velocidad, multitareas, prefieren lo gráfico-visual, amantes de los juegos, mas no de lo académico. Esta generación sugiere unos componentes en la identidad del individuo. Al respecto, las psicólogas Turkle (1995) y Wallace (1999) advierten que la red se está convirtiendo en un inmenso laboratorio de experimentación de identidad. En estos términos, el estudio sobre el uso de las TIC en Chile, explica el concepto de NATIVO DIGITAL así:

".....aquellos "teens" que nacieron cerca de un computador personal o un Nintendo, que han visto los mouses y teclados desde pequeños y aprendieron a interactuar con ellos naturalmente, los que

Figura 3. NTIC y semiosis en el contexto educativo

\begin{tabular}{|c|l|}
\hline Formalismo & Implica previsión y planificación de las acciones. Favorece la toma de conciencia y la autorregulación \\
\hline \multirow{5}{*}{ Interactividad } & $\begin{array}{l}\text { Posibilidades que ofrecen las TIC de que el estudiante establezca una relación contingente } \\
\text { e inmediata entre la información y sus propias acciones de búsqueda y procesamiento. } \\
\text { Permite una relación más activa y contingente con la información. Potencia el protago- } \\
\text { nismo del aprendiz. Facilita la adaptación a distintos ritmos de aprendizaje. Tiene efectos } \\
\text { positivos para la motivación y la autoestima. }\end{array}$ \\
\hline Dinamismo & $\begin{array}{l}\text { Ayuda a trabajar con simulaciones de situaciones reales. Permite interactuar con realidades } \\
\text { virtuales. Favorece la exploración y la experimentación. }\end{array}$ \\
\hline Multimedia & $\begin{array}{l}\text { Capacidad de los entornos basados en TIC para combinar e integrar diversas tecnologías. } \\
\text { Permite la integración, la complementariedad y el tránsito entre diferentes sistemas y for- } \\
\text { matos de representación (lengua oral y escrita, imágenes, lenguaje matemático, sonido, } \\
\text { sistemas gráficos, etc.). Facilita la generalización del aprendizaje. }\end{array}$ \\
\hline Hipermedia & $\begin{array}{l}\text { Resultado de la convergencia de la naturaleza multimedia del entorno más la utilización de } \\
\text { una lógica hiper-textual. Comporta la posibilidad de establecer formas diversas y flexibles } \\
\text { de organización de las informaciones, estableciendo relaciones múltiples y diversas entre } \\
\text { ellas. Facilita la autonomía, la exploración y la indagación. Potencia el protagonismo del } \\
\text { aprendiz. }\end{array}$ \\
\hline Conectividad & $\begin{array}{l}\text { Permite el trabajo en red de agentes educativos y aprendices. Abre nuevas posibilidades } \\
\text { al trabajo grupal y colaborativo. Facilita la diversificación, en cantidad y calidad, de las } \\
\text { ayudas que los agentes educativos ofrecen a los aprendices. }\end{array}$ \\
\hline
\end{tabular}

Fuente: Coll (2005) 
no conciben ir a revelar un rollo para ver los fotografías que tomaron, los que consideran una pérdida de tiempo escribir una carta y enviarla por el correo tradicional" (Africano, L. 2007).

El estudio estadístico realizado en Chile (Cárcamo, L. 2007) aclara que según el informe WIP World Internet Project (2006), la herramienta Fotolog se convierte en la preferida por los usuarios nativos digitales. Es la relevancia de la selección de imágenes a partir de los administradores de imágenes, con usuario femenino recurrente. Chile, Brasil, México son los países usuario que encabezan la tendencia de esta herramienta. En otras regiones de América Latina y del mundo se prefiere Hi5, Myspace o Flickr. La investigación que desarrolla Cárcamo, demuestra que en Chile el uso de Internet se concentra en poblaciones jóvenes, y que el Fotolog y el Weblog son espacios de cibervoyersimo y de una necesidad intrínseca de extrapolación de identidad individual. (Cárcamo, 2007)

Identidad individual va de la mano con el concepto de interacción. Aquí es necesario retomar al clásico Gofmann, quien advierte, al referirse a los usuarios en una interacción, que

"... cuando un individuo o actuante representa el mismo papel para la misma audiencia en diferentes ocasiones, es probable que se desarrolle una relación social. Al definir el rol social como la promulgación de los derechos y deberes atribuidos a un status dado, podemos añadir que un rol social implicará uno o más papeles, y que cada uno de estos diferentes papeles puede ser presentado por el actuante en una serie de ocasiones ante los mismo tipos de audiencia o ante una audiencia compuesta por la mismas personas." (Goffman, 1981: 28).

Junto a este escenario, incursionan aproximaciones conceptuales de las TIC en el contexto educativo, como las que destaca el argentino
Rubén Martínez, quien toma como punto de partida la mediación tecnológica para caracterizar la mediación en el proceso enseñanza-aprendizaje. Obsérvense las figuras 4 y 5 , en donde se visualiza la mediación a partir de la clase de relación entre SUJETOS \& OBJETOS.

Figura 4. La relación mediada Herramienta mediadora

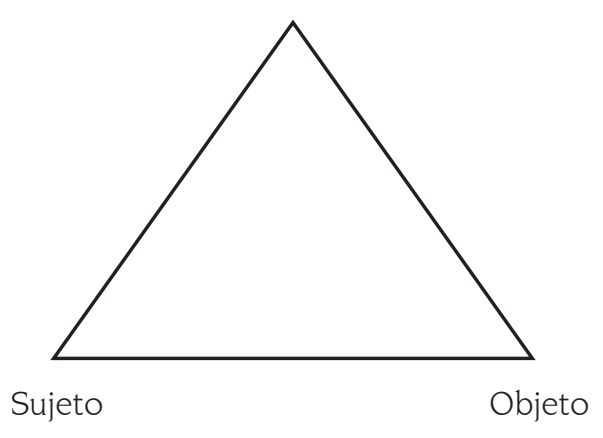

Relación mediada por herramientas/aparatos/ instrumentos. Este gráfico explica que la relación básica entre sujeto $\varepsilon$ objeto, no es directa, por ello la línea punteada (Martínez, 2006).

En la figura 5, el tejido de relaciones es más variado: el sujeto interactúa con sujetos colectividad con el propósito de llevar a cabo una actividad a partir del trabajo compartido, colaborativo. En términos de Martínez, este gráfico ilustra las relaciones en el proceso de enseñanza-aprendizaje en contexto de interacción en línea.

Figura 5. Sujetos y Relaciones colaborativas

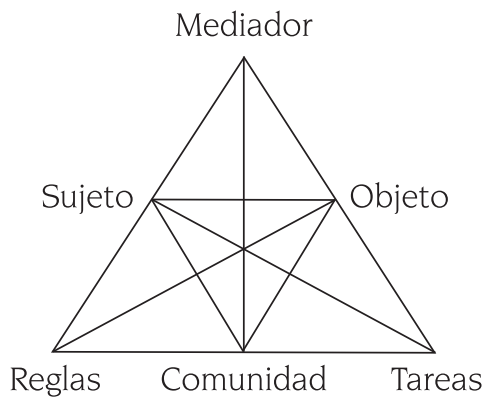

Fuente: Autoría propia. 


\section{Formas y significantes en TIC}

La e-sociedad, la sociedad de lo multimediático, la que está "mediada" por las mediaciones de las TIC, ya nos permite -con ciertas licencias- referirnos a las nuevos actores digitales en términos tales como la generación chat, los jóvenes messenger.

La incursión de contextos emergentes comunicacionales que atraviesan la conexión nodal entre uno o varios y simultáneos sujetos sugiere, a la vez, revisar en detalle la incursión, a la vez, de significantes -materializaciones del signo, en términos de Saussure--, tal y como lo explica Racioppe:

"Cuando chatean es como si hablaran y en ese diálogo aparecen marcas identitarias, qué digo de mí, cómo me defino, cómo me narro a los otros da cuenta de cómo me percibo y cómo quiero que me perciban. Además, el espacio del chat tiene ciertos códigos y elementos que permiten conocer los estados de ánimo del otro, los tonos en que está "hablando". Los emoticones, cada vez más variados, son uno de esos elementos, el uso de mayúsculas, de signos de exclamación son todas claves que permiten descifrar cuán en serio dice lo que dice el otro, anclar los sentidos de su discurso. Estos códigos del chat han nutrido los mensajes de texto y viceversa: las abreviaturas para economizar tiempo y espacio, las letras usadas por fonética (T kiero, ksa, bso.) han modificado los modos de escribir y han creado reglas propias para el chat y los mensajes de texto; reglas que son verdaderas recreaciones gramaticales" (Racioppe, 2008: p.9 ).

Más aún, para quienes son fieles a la gramática pura, nos advierte la misma autora: "Lejos de asustarnos o pregonar la "destrucción" del castellano, deberíamos comprender la creatividad para crear nuevos registros y entender que quizá una de nuestras tareas como educadores sea enseñarles a darse cuenta de los contextos para usar esos registros" (Racioppe, 2008: p. 10).

\section{Nuevos objetos de estudio y etnografía virtual}

En este cibercontexto no se pueden descuidar los abordajes metodológicos. El abordaje de signos textuales, sonoros y audiovisuales que fluyen en el ciberespacio -entendido como el espacio de interacción posibilitado por redes de ordenadores (Hine, 2004: 193)- obliga a la etnografía a hacer inmersión desde las nuevas relaciones entre lo observado y el observador. Se debe delimitar la unidad de observación en estos contextos cibernéticos. Por tanto, es sustancial caracterizar el contexto en que fluyen y se interconectan estas nuevas realidades objeto de estudio. Mayans (2002), arguye 5 rasgos de un contexto ciberespacial, visualizados en el siguiente gráfico (Figura 6):

Figura 6. Rasgos del Ciberespacio

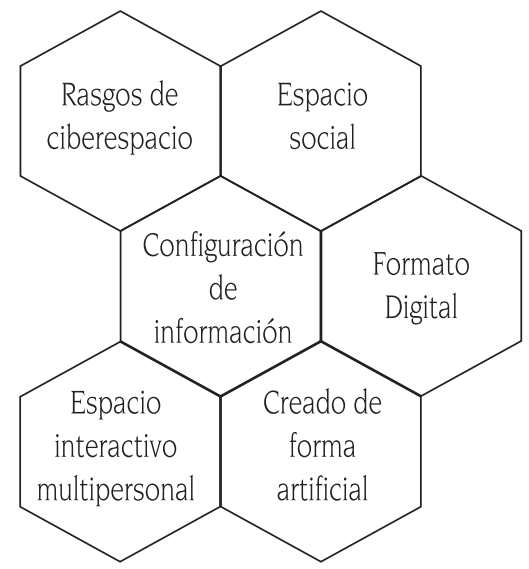

Fuente: Autoría propia

En medio de este contexto, se construyen, se producen y se interpretan sistemas simbólicos en tiempos y espacios "online" E "offline". En este sentido, también es relevante delimitar y comprender los usos y las prácticas de estos sistemas sígnicos-significantes en medio de estos dos sub-contextos. Para ello, es pertinente citar la caracterización que hace la experta Christine Hine, quien reconoce dos modalidades de interacción en tanto que son dos formas de ver la Internet: Internet como Cultura (online), Internet como artefacto (offline). 
La primera hace alusión al lugar donde se producen las interacciones que originan la formación de una cultura; allí se analiza el contexto y los discursos de sus participantes. Lo online son vínculos que se sostienen en el ciberespacio, son los usos que las personas hacen de la tecnología.

La segunda interacción se refiere al producto de la cultura, con un significado según su uso, con representaciones mediáticas; hace alusión a los contactos cara a cara en el espacio físico, personas con rasgos etnográficos concretos, con metas y gustos contextualizados. Entre ambos escenarios se crean múltiples y variadas referencias materiales y simbólicas. Sugerimos la convergencia de estos dos sub-contextos, tal y como se aprecia en la figura 7.

La experta Hine, en sus investigaciones desde la etnografía virtual, orienta conceptos y rutas bastante viables en el abordaje de estas nuevas realidades investigativas, propias de la sociedad mediática del siglo XXI.
Estamos de acuerdo en que es prioritario avanzar en investigaciones cuyo enfoque sea el de las interacciones offline, en tanto es en este tipo de productos comunicativos nodales en donde se construyen los sentidos de los grupos étnicos. La mediación a través de internet producto supera sus características técnicas y pasa a un primer lugar: la invención de significados socioculturales en tanto que es la inventiva de la gente quien confiere dicha semiosis, dicha construcción simbólica.

\section{Junto a las TIC, lo educativo}

Dentro del espectro de estudios que abordan la necesidad de incorporar el uso del computador -como elemento de las NTIC- no como accesorio sino como un dispositivo protagónico en los procesos de enseñanza-aprendizaje, se deben mencionar autores como Ríos Ariza y otros (2000), Chiecher (2010), Martínez y otros (2011), Dibut (2010), López (2001) , Fazeli y otros (2000); todos investigadores que destacan el papel crucial del uso del computador al permitir implementar la

Figura 7. Sub-contextos en la construcción de símbolos ${ }^{1}$
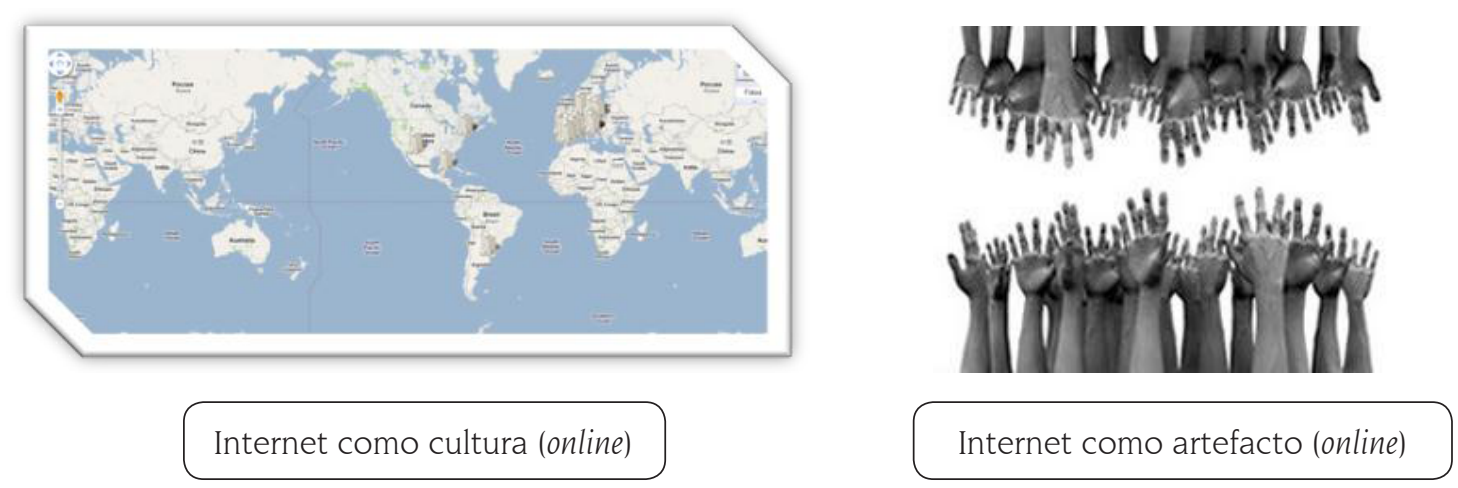

Fuente: Autoría Propia

1 Las fotografías de la figura 7 fueron consultadas en: http://maps.google.es $/$ maps $/ \mathrm{ms}$ ?ie=UTF8Eoe =UTF8Emsa =0Emsid= 1079 35177796040719442.000434e5fb9710092cf48

http://www.google.com.co/imgres?imgurl=http://1 bp.blogspot.com/_7Z4PYJBym5o/S7MSBMk3O8I/AAAAAAAAAYo/TdH19iGlc]8/S1600-R/adolescentes_\%2Bmanos7.jpgEimgrefurl=http://adolescentesgeneracionnet.blogspot.com/Eusg=_ DRD0Vls0a8

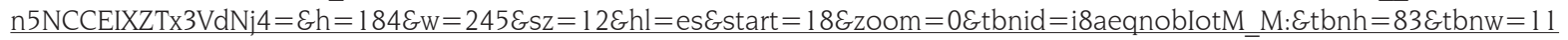
0Eei=BMcXTVSFH4-cgQe51Z0OEprev=/search\%3Fq\%3Dgeneracion\%2Bnet\%26um\%3D1\%26hl\%3Des\%26rlz\%3D1R2SKPT_es CO426\%26biw\%3D1280\%26bih\%3D549\%26tbm\%3DischEum = 1 Eitbs $=1$ \&iact $=$ hcEvpx $=617$ Evpy $=207 \varepsilon$ dur $=4119 \varepsilon$ hovh $=8$ $\underline{3 E \text { hovw }}=110 \mathcal{E} \mathrm{tx}=129 \mathrm{Ety}=49 \mathrm{Epage}=2 \mathcal{E} \mathrm{ndsp}=21 \mathrm{Eved}=1 \mathrm{t}: 429, \mathrm{r}: 17, \mathrm{~s}: 18 \mathrm{Ebiw}=1280 \mathrm{Ebih}=549$ 
alfabetización tanto informacional como digital. La generación del siglo XXI pasó del tablero tradicional a la pizarra digital interactiva, de la LAN (Local Area Net) a la BAN (Body Area Network), escenario familiar para los jóvenes universitarios: nativos digitales.

\section{Jóvenes y consumos de TIC, estudios de caso en América y Europa}

Los estudios que se presentan a continuación se organizaron en orden alfabético por país y por su recurrencia en este tópico investigativo: (Figuras 8, 9)

\section{Hacia nuevas pesquisas}

A pesar de las divergencias entre la sociología y la psicología cognitiva, es una realidad la existencia de la Generación Net. De hecho la existencia del observatorio en Argentina, donde se explora y analiza el uso de las TIC (UBA, 2010), recoge tendencias de este proceso comunicativo. La convivencia entre los nativos digitales y los inmigrantes digitales debe superar la brecha y propiciar puntos de encuentro a partir del Internet "offline" y "online". Sin embargo, creemos que más que estar afrontando una mediación de orden meramente tecnológico, sí es necesario indagar los impactos, los niveles de gratificación

Figura 8. Estudios en América y Europa

\begin{tabular}{|c|c|}
\hline País & Datos del estudio \\
\hline $\begin{array}{l}\text { ARGENTINA } \\
\text { El impacto de las TIC sobre la juventud: } \\
\text { metáfora y representación en ciencias } \\
\text { sociales } \\
\text { El consumo y uso de pantallas en los } \\
\text { Cibers de Rosario 2005-2007: }\end{array}$ & $\begin{array}{l}\text { Este estudio publicado en un portal argentino, especializado en jóvenes } \\
\text { y tics, toma como eje temático un elemento linguistico/semiotico: estu- } \\
\text { diar la metáfora es estudiar el significado de los procesos comunicativos } \\
\text { y de interacción, incluso involucra estudiar el proceso de significación. } \\
\text { El estudio advierte la existencia de } 2 \text { clases de metáforas: las vivas y las } \\
\text { muertas. Las primeras hacen alusión a significados consolidados en } \\
\text { diccionarios etimológicos; mientras que las segundas refieren al proceso } \\
\text { mismo de significación, dan información del mundo que se está creando, } \\
\text { este podría ser el caso de las metáforas creadas a partir de las TIC. (Feliu, } \\
\text { 2010) } \\
\text { La investigación recoge datos valiosos a partir del boom de WWW (Castro, } \\
\text { 2010). En la ciudad de Rosario-Argentina, a partir de la observación y de la } \\
\text { aplicación de entrevista intensiva a adolescentes y jóvenes se evidencia la } \\
\text { proliferación de entornos tecnológicos TIC que van desde el uso permanen- } \\
\text { te del e-mail, chat, foros, y messenger, todos en un escenario específico: } \\
\text { los cibercafés. Se mencionan aspectos tales como: uso lúdico de las TIC, } \\
\text { uso de interfaces, tales como: Celulares, PC, Ipod, MP3, usos que han y } \\
\text { siguen transformando las formas de contacto y de comunicación entre los } \\
\text { jóvenes. Lo interesante del estudio es la evidencia de un fenómeno que en } \\
\text { términos de Foucault (1984), siguen analistas como Stewart (1999), Nunes, } \\
\text { Finquelievich (2007), Maeso, Liff (2002): LA HETEROTOPÍA, "diversidad de } \\
\text { lugares que reflejan otros lugares" (Castro 2010), es el vínculo entre las } \\
\text { dicotomías: LO REAL / LO VIRTUAL; LO LOCAL/LO GLOBAL. Los ciberca- } \\
\text { fés anulan las diferencias sociales, son los "spaces of elsewhere" (Nunes } \\
\text { 2006), es el "espacio de los flujos" (Castells, 1998), y en este espacio de } \\
\text { flujos emergen las TICs. }\end{array}$ \\
\hline
\end{tabular}

(continúa) 
Figura 8. Estudios en América y Europa (continuación)

\begin{tabular}{|c|c|}
\hline País & Datos del estudio \\
\hline \multirow[t]{2}{*}{$\begin{array}{l}\text { COLOMBIA } \\
\text { Acceso, conocimiento y uso de las herra- } \\
\text { mientas especializadas de Internet entre } \\
\text { la comunidad académica, científica, } \\
\text { profesional y cultural de la Universidad } \\
\text { de Antioquia } \\
\text { Interacciones comunicativas en un } \\
\text { entorno virtual de aprendizaje": }\end{array}$} & $\begin{array}{l}\text { La investigación propone un modelo de gestión de conocimiento a partir } \\
\text { del uso de las TIC para aplicarse en la Universidad de ANTIOOUIA. La } \\
\text { investigación menciona a uno de los teóricos clásicos sobre la temática } \\
\text { de las TIC: SOCIEDAD RED "Esta es la nueva estructura social de la era } \\
\text { de la información, que denomino la sociedad red porque está compuesta } \\
\text { por redes de producción, poder y experiencia, que construyen una cultura } \\
\text { de la virtualidad en los flujos globales que transcienden el tiempo y el } \\
\text { espacio" (Castells, 1998). Junto a esta caracterización, resulta pertinente } \\
\text { destacar los } 4 \text { tipos de brechas digitales en que dinamiza el mundo de la } \\
\text { información: la brecha digital internacional (comparación entre países), la } \\
\text { brecha digital doméstica (entre regiones, localidades, grupos sociales), la } \\
\text { brecha digital individual, la brecha digital organizacional (Uribe, et all, 2004) }\end{array}$ \\
\hline & $\begin{array}{l}\text { Esta investigación realizada en la Universidad de Medellín, es de orden } \\
\text { cualitativo, brinda insumos tanto conceptuales como metodológicos en } \\
\text { la indagación del entorno virtual, utilizando el análisis del discurso en la } \\
\text { interpretación de datos (Vásquez, et al, 2009). }\end{array}$ \\
\hline $\begin{array}{l}\text { COSTA RICA } \\
\text { El uso de la tecnología de la información } \\
\text { y el diseño curricular" }\end{array}$ & $\begin{array}{l}\text { Este estudio (Gómez, À, 2008), es valioso dada la convergencia que aborda } \\
\text { entre TIC y Currículo; específicamente en la gestión curricular. El hallazgo } \\
\text { relevante sustenta la necesidad de diseñar una propuesta desde } 3 \text { ángulos: } \\
\text { el pedagógico, el académico y el administrativo. Los } 3 \text { son necesarios para } \\
\text { abordar esta realidad mediada a través de las TIC. }\end{array}$ \\
\hline \multirow[t]{2}{*}{$\begin{array}{l}\text { ESPAÑA } \\
\text { Consum de les TIC per part del joves en } \\
\text { espais públics et privats de lleure: } \\
\text { Integración de las TIC en la Univer- } \\
\text { sidad": }\end{array}$} & $\begin{array}{l}\text { Estudio que compila los resultados de la primera fase. Se registra la ten- } \\
\text { dencia de entrevistas realizadas en cibercafés a adolescentes en Barce- } \\
\text { lona (Gil-Lajeunesse, 2003). Los aspectos que se indagan a través de los } \\
\text { instrumentos utilizados buscan información sobre la Interacción "offline" } \\
\text { (Cibercafés), la apropiación del espacio, la apropiación simbólica, el uso } \\
\text { de otras tecnologías, el propósito de la conexión y uso de tiempo, habi- } \\
\text { lidades tecnológicas desarrolladas o requeridas, la identidad, y sobre la } \\
\text { interacción en línea. }\end{array}$ \\
\hline & $\begin{array}{l}\text { Entre la economía de mercado y la innovación pedagógica. Este trabajo } \\
\text { indaga en torno a } 3 \text { escenarios: estudios de caso pedagógicos, cuestiona- } \\
\text { rio en línea para el docente y una propuesta de foro en línea sobre TIC e } \\
\text { impacto. Se menciona el grado de afectación que han causado las TIC en el } \\
\text { sujeto, en su relación con el conocimiento, y en el entorno socio-cultural } \\
\text { del individuo (García, 2007). Se ha transformado tanto la forma de repre- } \\
\text { sentar como la forma de interpretar, comenta el profesor uruguayo García. } \\
\text { A partir de estos supuestos, el estudio que presenta Baute, versa sobre la } \\
\text { relación de las necesidades del mercado laboral frente a la incorporación } \\
\text { de las TIC, mencionando que a partir del cambio en las representaciones } \\
\text { (proceso de semiosis), surge a la vez el cambio en roles tanto del estudiante } \\
\text { como del docente. Advierte que no es en el nivel de vanguardia sobre las } \\
\text { TIC con que disponga la universidad donde yace lo innovador, sino en el } \\
\text { USO PEDAGÓGICO que se da a esta tecnología. (Baute, 2008) }\end{array}$ \\
\hline
\end{tabular}


Figura 9. Estudios en América y Europa

\begin{tabular}{|c|c|}
\hline País & Datos del estudio \\
\hline $\begin{array}{l}\text { ESTADOS UNIDOS } \\
\text { Convenience, Communications, } \\
\text { and Control: How Students Use } \\
\text { Technology }\end{array}$ & $\begin{array}{l}\text { El e-book compila un nutrido estudio de casos sobre le generación NET en } \\
\text { el contexto de la sociedad norteamericana. El estudio realizado por Kravik, } \\
\text { recoge la respuesta de una población de } 4.374 \text { estudiantes - } 95 \% \text { de los } \\
\text { jóvenes tienen } 25 \text { años-, en } 13 \text { instituciones y en } 5 \text { estados. De este estu- } \\
\text { dio destacamos los siguientes datos: "Students reported using computers } \\
\text { for writing documents ( } 99.5 \text { percent) and e-mails ( } 99.5 \text { percent), followed by } \\
\text { surfing the Internet for pleasure ( } 97.2 \text { percent) and for classroom activities } \\
\text { (96.4 percent). Students reported using technology for creating/editing video } \\
\text { and audio and for creating Web pages the least" (Oblinger, 2005, p.84). Estos } \\
\text { datos nos evidencian los altos niveles de uso de TIC tanto en lo académico } \\
\text { como en lo lúdico. }\end{array}$ \\
\hline $\begin{array}{l}\text { MÉXICO } \\
\text { Diagnóstico en el uso de las TIC de } \\
\text { los estudiantes de primer ingreso a } \\
\text { nivel superior en la Universidad de } \\
\text { Colima: }\end{array}$ & $\begin{array}{l}\text { El estudio plantea un conjunto de propuestas que la universidad debe em- } \\
\text { prender, tomando como marco referencial el manejo básico del computador } \\
\text { y de la internet por parte de los estudiantes de primer semestre en U. de } \\
\text { Colima. En este estudio se aborda tanto al docente como al estudiante, cuyo } \\
\text { alcance sugiere la necesidad de diseñar e incorporar en los programas, acti- } \\
\text { vidades que involucren el uso de las TIC e implementar el uso de portafolios } \\
\text { electrónicos tanto en alumnos como en docentes. (Sánchez, et al, 2006). }\end{array}$ \\
\hline
\end{tabular}

y usos que esta generación en el contexto educativo puede potenciar a partir del reconocimiento de la mediación simbólica-cultural. Ya uno de los directores de la RAE, Real Academia Española, expresa, ante el uso de la TIC y su afectación en la lengua española que "estas nuevas formas de expresión lingüística, como abreviar palabras o usar letras para reemplazar vocablos, no afectarán de manera importante a la lengua", puesto que las personas "sabemos distinguir perfectamente el nivel y el medio en el que nos comunicamos" (La Nación, 2010). Vale la pena preguntarse si la generación net y la multitasking piensan lo mismo.

\section{Bibliografía}

ADELL, Jordi (1997), "Tendencias en educación en la sociedad de las tecnologías de la información", consultado el 22 de junio de 2011, en: http://www. uib.es/depart/gte/revelec7.html
AFRICANO, L., (2007) "Niños digitales: Las marcas se preparan para el cambio", en Revista Digital Infobrand, Chile, consultado el 2 de junio de 2011, en: http://www.infobrand.com.ar/nota.php? $i d x=9299$

AGUADO, T- HERRAZ, MERCEDES (2006), Mediación Social Intercultural en el Ámbito Socioeducativo, consultado el 16 de junio de 2011, en: http://redalyc. uaemex.mx/redalyc/pdf/1610/161016087001.pdf

ARAUJO, I. (2002). Mediaciones y poder, en Orozco Gomez, G.(2002) Recepciones y mediaciones. Edit. Norma. Bs.As.

BAUTE, M Benjamin (2008), "La integración de las TIC en la universidad: Entre la economía de mercado y la innovación pedagógica", consultado el 25 de mayo de 2011, en http://dugi-doc.udg.edu/bitstream/10256/894/1/118.pdf

CÁRCAMO, Luis (2007), "Nativos Digitales Chilensis: Los jóvenes, al sur de la Internet", consultado el 
10 de junio de 2011, en : http://www.revistalatinacs. org/_2008/03_Carcamo_y_Nesbet.html

CASTELLS, M. y otros (1986), El desafío tecnológico. España y las nuevas tecnologías, Alianza Editorial, Madrid

CASTELLS, Manuel (1998). La era de la información. Economía, sociedad y cultura.Vol. La Sociedad Red. España: Alianza Editorial.

CASTRO R., Sebastián (2010), "El consumo y uso de pantallas en los Cibers de Rosario 20052007", consultado el 23 de junio de 2011, en: http://www.perspectivasdelacomunicacion.cl/ revista_2_2010/01texto.pdf

CROVI DRUETTA, Delia (2000), Tecnología satelital para la enseñanza, Instituto Latinoamericano de la Comunicación Educativa (ILCE), México

CHIECHER, Analía (2010), Interacciones entre alumnos en aulas virtuales: Incidencia de distintos diseños instructivos, Revista de Medios y Educación, consultado el 4 de junio de 2011, en: http://intra. sav.us.es:8080/pixelbit/images/stories/a10_0038premaq.pdf

COLL, C. (2005). Psicología de la educación y prácticas educativas mediadas por las tecnologías de la información y la comunicación: una mirada constructivista. Sinéctica, (25), Separata, 1-24.

DÍAZ-BARRIGA, F. (2005), "Principios de diseño instruccional de entornos de aprendizaje apoyados con TIC: un marco de referencia sociocultural y situado", consultado el 13 de junio de 2011, en: http://investigacion.ilce.edu.mx/tyce/41/art1.pdf

Dibut, et all (2010), Las nuevas tecnologías de la información y la comunicación como mediadoras del proceso de enseñanza-aprendizaje, consultado el 20 de mayo de 2011, en: http://laurusita.wordpress. com/2010/01/02/las-nuevas-tecnologias-de-la-informacion-y-la-comunicacion-como-mediadorasdel-proceso-de-ensenanza-aprendizaje/

DUBOIS, J. (1998), Diccionario de Lingüística, Madrid, Alianza Editorial.
FAINHOLC, Beatriz (2004), El concepto de mediación en la tecnología educativa apropiada y crítica, consultado el 25 de junio de 201 1, en: http://cmapsinternal.ihmc.us/rid = $1119466861556 \_1804172076$ 502/educ.ar\%20\%20Educacion\%20y\%20TIC_\%20 El\%20concepto\%20de\%20mediacion\%20en\%20 la\%20tecnologia\%20educativa\%20apropiada\%20 \%20critica.pdf

FAZELI, H., Manzano, V. y Pérez, F. (2000). Variables contextuales en el éxito de las aplicaciones multimedia en la Universidad. Revista Electrónica de Metodología Aplicada, consultado el 12 de mayo de 2011, en: http://www.psico.uniovi.es/REMA/ v5n1/al/

FEIXA, C. et al. (2002). "Estils de vida i identitat". En: GÓMEZ-GRANELL, C.; GARCIA-MILÀ, M.; RIPOLMILLET, A.; PANCHÓN, C. (coord.). La infància i les famílies als inicis del segle XXI. Informe 2002 [en línea]. Barcerlona: Instituto de Infancia y Mundo Urbano. Observatorio de la Infancia y la Familia

FELIU, Joel (2010), El impacto de las TIC sobre la juventud: metáfora y representación en ciencias sociales, consultado el 9 de junio de 2011, en: http://www. perio.unlp.edu.ar/revistadejuventud/?q=node/52

FINQUELLEVICH- Prince (2007), El (Involuntario) Rol social de los cibercafés, consultado el 2 de junio de 2011, en: http://www.oei.es/tic/rolciber cafes.pdf

FOUCAULT, Michel (1984), Des espaces autres. Hétérotopies, consultado el 5 de junio de 2011 , en: http:// foucault.info/documents/heteroTopia/foucault. heteroTopia.fr.html

GARCÌA-TESKE, E. (2007), Los discursos sobre las nuevas tecnologías en contextos educativos, consultado el 20 de junio de 2011, en; http://www. rieoei.org/deloslectores/1637Teske.pdf

GIL, et al. (2003), ¿Nuevas tecnologías de la información y la comunicación o nuevas tecnologías de relación? Niños, jóvenes y cultura digital, consultado el 18 de junio de 2011, en: http://www.uoc.edu/ dt/20347/index.html 
Revisión de la gramatica emergente: lenguajes, jovenes y mediaciones tic *

GIL-LAJEUNESSE (2003), Consum de les TIC per part del joves en espais públics et privats de lleure, consultado el 30 de mayo de 2011, en

GOFFMAN, E. (1981). La presentación de la persona en la vida cotidiana. Buenos Aires: Amorrortu Editores

GÒMEZ, A. Gisselle ( 2008), El uso de la tecnología de la información y el diseño curricular, consultado el 12 de junio de 2011, en: http://redalyc.uaemex. $\mathrm{mx} / \mathrm{pdf} / 440 / 44032107 . \mathrm{pdf}$

GUTIÉRREZ, María; Ball, M.; Márquez, E. (2008), "Signo, significado e intersubjetividad: una mirada cultural", consultado el 9 de junio de 2011, en: http:// redalyc.uaemex.mx/pdf/356/35614570004.pdf

GRUFFAT, C (2004), Generación M: los chicos que crecieron con los nuevos medios, consultado el 6 de julio de 2011, en: http://scholar.google.com.co/sch olar?q=sherry+TURKLE+NATIVOS+DIGITALES+ 1995Ehl=esEas_sdt=0Eas _ vis $=1 \mathrm{Eoi}=$ scholart

HERRERA-BATISTA, Miguel Á. Universidad Autónoma Metropolitana-Azcapotzalco, México http://www. rieoei.org/deloslectores/2630Batistav2.pdf

HINE, Christine (2004), Etnografía virtual, UOC, Barcelona.

ILVAY, Serafin (2000), Nuevos escenarios y tecnologías de la comunicación en el umbral del tercer milenio, consultado el 24 de junio de 2012, en: http://www. razonypalabra.org.mx/anteriores/n18/18silvay.html

LA NACIÓN, "El lenguaje utilizado en chats y celulares no afectaría el idioma", consultado el 3 de julio en: http://www.lanacion.com.ar/03/10/22/dg _ 537528. asp

LIFF, S, Steward, Fy Watts, P (2002), "New public places for Internet access: networks for practices-based learning and social inclusion", en Steve Woolgar (ed). Vitual society? Technology, cyberbole, reality, Oxford University Press

LÓPEZ, Alfonso (2001), Documentación digital y nuevas tecnologías de la información, consultado el 10 de mayo de 2011, en: http://multidoc.
rediris.es/cuadernos/num13/talleres/pdf/Radio YEPES.pdf

MARTINEZ, Rubén Darío (2006), TIC en la Educación: Sobre las unidades de análisis, consultado el 30 de mayo de 2011, en. www.niee.ufrgs.br/eventos/ RIBIE/2006/ponencias/art084.pdf

MARTínEZ, Juan C. (2011), Uso de las Nuevas Tecnologías en los centros educativos, consultado el 28 de mayo de 2011, en: http://www.publicatuslibros.com/fileadmin/Biblioteca/Libros/Tecnicos/ Uso_de _las_nuevas_tecnologias_en _los _ centros_educativos.pdf

MAYANS, J. (2002): Nick CiberEspacio /set topic conceptos y términos para el análisis socioantropólogico. I Congreso ONLINE del Observatorio para la CiberSociedad. Comunicaciones-Gr upo $10 \mathrm{El}$ investigador en la sociedad digitalizada. Consultado el 25 de mayo de 2011, en: http://cibersociedad. rediris.es/congreso/comms/g10mayans.htm

MÉNDEZ, Miguel (2005), El Impacto de las Nuevas Tecnologías de la Información en la Sociedad y su Cultura Científico-Tecnológica, consultado el 13 de junio de 2012, en: http://www.razonypalabra.org. mx/anteriores/n43/mmendez.html

MOCiÑO, José. Año 2000. "Integración y Exclusión social debido al lenguaje". Disponible en http:// es.scribd.com/mobile/documents/43818977/downl oad?commit=Download+NowEsecret _ password, consultado el 10 de marzo de 2011

MORDUCHOWICZ, Roxana (2008), Generación Multimedia, Significados, consumos y prácticas culturales de los jóvenes, Paidós

NUNES, Mark (2006), Ciberspaces of Everyday Life, Minessota, Electronic

NÚÑEZ P. Sonia (2005). Género y televisión. Estereotipos y mecanismos de poder en el medio televisivo. Comunicar: Revista científica iberoamericana de comunicación y educación.

OBLINGER \& Oblinger, (2005), Educating the Net Generation, consultado el 1 de Julio de 2011, 
en: http://net.educause.edu/ir/library/pdf/pub 7101.pdf

PISCITELLI A. (2009), Nativos Digitales: Dieta cognitiva, inteligencia colectiva y arquitecturas de participación, Aula XXI-Santillana,

RACIOPPE, Rotouno, Poiré, Porta (2008), Jóvenes y Tics. Modos de socialización y construcción de identidad (es), consultado el 10/03/2011 en http:// www.ucasal.net/unid-academicas/artes-y ciencias/ congresos/redcom10/archivos/redcom-ponencia/ Ejes23y4/Eje3/Mesa3-2/Racioppe-Rotouno-PortaPoire.pdf

REGUILLO CRUZ, Rossana. "Emergencia de culturas juveniles, Estrategias del desencanto", Buenos Aires, Norma, 2000.

RÍOS A, Manuel- Cebrian (2000), Nuevas tecnologías de la información y de la comunicación aplicadas a la educación, Publicación Málaga : Ediciones Aljibe

ROSARIO, Jimmy, 2005, "La Tecnología de la Información y la Comunicación (TIC). Su uso como Herramienta para el Fortalecimiento y el Desarrollo de la Educación Virtual". Disponible en Observatorio para la CiberSociedad en http://www.cibersociedad.net/archivo/articulo.php?art=218, consultado el 20 de marzo de 2011

ROSE, N. (1996). Inventing ourselves. Nueva York: Cambridge University Press, 1998

RIVERO, I. (2001). Una aproximación crítica a las prácticas psicoterapéuticas: vicisitudes de un viaje a través del análisis del discurso. Trabajo de Investigación. Barcelona: Departamento de Psicología Social. Universidad Autónoma de Barcelona.

. (2002). Cuando las subjetividades son el desajuste tecnológico: algunas reflexiones acerca de la psicoterapia y la artifactualidad de "su" objeto. Barcelona: IX. Congreso de Antropología.

SÁNCHEZ, A. et al, (2006) Diagnóstico en el uso de las TIC de los estudiantes de primer ingreso a nivel superior en la Universidad de Colima,
TORREBLANCA N., Omar;Rojas-Drummond, Sylvia, "Mediación tecnológica para el desarrollo de habilidades de observación en estudiantes de Psicología: un enfoque socioconstructivista", consultado el 17 de junio de 2011, en: http://redalyc. uaemex.mx/pdf/132/13211845004.pdf

THOMPSON, John (1998). Los media y la modernidad Una teoría de los medios de comunicación, Barcelona, Paidós Comunicación

TURKLE (1995), Inmigrantes Digitales \& Nativos Digitlaes, consultado el 28 de mayo, en: http://scholar. google.com.co/scholar?q=sherry+TuRKLE+NATI VOS+DIGITALES+1995Ehl=esEas _ sdt=0Eas vis $=1$ Eoi $=$ scholart

URIBE, et all, (2007), "Acceso, conocimiento y uso de Internet en la Universidad de Antioquia: modelo de diagnóstico y caracterización", consultado el 3 de mayo de 2011, en: http://redalyc.uaemex.mx/ redalyc/pdf/1790/179014342002.pdf

UBA (2010) Observatorio del uso de las TICS, Universidad de Buenos AIRES, http://www.delconocimiento.com.ar/?cat=4

VALLIN, M (2010), Presentación Alejandro Piscitelli: Nativos Digitales, consultado el 4 de junio de 2011, en: http://www.slideshare.net/MonVall/ppointnativos-final

VÀSOUEZ, Claudia, et all, (2009), "Interacciones comunicativas en un entorno virtual de aprendizaje", consultado el 8 de junio de 2011, en: http://www. colombiadigital.net/newcd/dmdocuments/222. interacciones\%20comunicativas.pdf

WALLACE, P. (1999) The Psychology of the Internet, New York: Cambridge University Press

WIP, World Internet Project, (2006), consultado el 3 de julio de 2011, en: http://comunicaciones. uc.cl/prontus fcom/site/artic/20080418/asocfile/20080418230431/wip_chile_2006_in _ forme_final_en_pdf_ok.pdf 